\title{
SAE TECHNICAL

\section{Ceramic Foams as Catalyst Substrates: Pre-catalyst Application Homogenising the Exhaust Flow upstream of Aftertreatment Devices}

\author{
P. Dimopoulos, C. Bach \\ Empa, Swiss Federal Laboratories for Materials Testing and Research, Laboratory for I.C. Engines \\ U. F. Vogt \\ Empa, Swiss Federal Laboratories for Materials Testing and Research, \\ Laboratory for High Performance Ceramics \\ K. Herrmann \\ Aerothermochemistry and Combustion Systems Laboratory ETH, Swiss Federal Institute of Technology
}




\title{
Ceramic Foams as Catalyst Substrates: Pre-catalyst Application Homogenising the Exhaust Flow upstream of Aftertreatment Devices
}

\author{
P. Dimopoulos, C. Bach \\ Empa, Swiss Federal Laboratories for Materials Testing and Research, Laboratory for I.C. Engines \\ U. F. Vogt \\ Empa, Swiss Federal Laboratories for Materials Testing and Research, Laboratory for High Performance Ceramics \\ K. Herrmann \\ Aerothermochemistry and Combustion Systems Laboratory ETH, Swiss Federal Institute of Technology
}

Copyright @ 2007 SAE International

\begin{abstract}
Non-homogeneities in the exhaust line regarding flow distribution and mixing of exhaust gases upstream of catalytic converters or particulate filters are a major source of conversion efficiency reduction due to partial volume utilization.

Current supports for catalytic converters use a honeycomb monolithic substrate with only a limited potential for increased wall contacts of the gas molecules due to their laminar channel flow profile. Non homogeneities of the flow distribution at the entering cross section of the catalytic converter prevail also inside the converter channels since no momentum exchange is possible perpendicular to the main flow direction.

The ceramic based foams developed and patented by Empa are a promising alternative. In the upstream of exhaust aftertreatment devices they ceramic foams redistribute the exhaust gases homogenizing the flow, enhancing turbulence and species mixing, without increasing flow resistance to prohibitive levels. Catalytically coated, they even can initiate, facilitate or even complete oxidation of unburnt $\mathrm{HCs}$ and $\mathrm{CO}$ and oxidize a substantial fraction of particulates while generating required species for the following aftertreatment step.

In this paper we present some material development aspects as well as flow field investigations performed in the up and the downstream of the ceramic foams, demonstrating their potential.
\end{abstract}

\section{INTRODUCTION}

The homogeneity of flow distribution in catalytic converters is a major parameter determining conversion effi- ciency, thermal load and thus useful life. Non homogeneities and asymmetries upstream of the catalytic converter, as well as flow recirculation zones at symmetric conical inlets can lead to significant non-uniformity of the flow inside the monolith. Flow non-uniformity results in significant differences in ignition characteristic, thermal load and conversion efficiency among the channels. Several studies have provided a series of relevant results, [1, 2, 3, 4 and 5] mostly by CFD simulations of the flow field as well as of the chemical reactions upstream and inside the catalyst channels. All studies report that uneven distribution at the inlet can strongly affect catalytic converter performance, and lead to high thermal gradients in the monolith also strongly affecting light-off during the warm-up phase.

The flow field in a monolith is a result of the pressure distribution in the upstream section determined by the flow features there. In addition, it is also strongly affected by the pressure distribution on the inlet face of the monolith, which in turn is determined by the frictional losses inside the monolith channels. The frictional pressure drop in a given channel is a monotonic function of the mass flux and viscosity of the fluid in the channel. Channels with lower mass flux offer lesser frictional resistance than channels with higher mass flux. Thus the flow would immediately be directed more towards the channels with lower frictional losses. The friction in the channels tends to make the flow (and the pressure drop) uniform across the cross-section of the monolith. However, the mass flow distribution is determined through a balance between the effect of friction and convective forces upstream of the monolith. Studies [1-5] give comprehensive examples of the non-symmetrical flows and their consequences for the catalyst operation. A com- 
prehensive overview of main flow and chemical reaction features in catalysts is given in [6].

Flow analysis is even more complex as important in diesel particulate filters (DPF). To some extent the flow distribution inside a DPF should be self regulating during the loading, at least, when starting from a clean or completely regenerated filter. On the other hand frictional effects inside the DPF channels are weak; DPFs are usually designed with larger channels than catalysts in order to compensate for the additional, significantly higher, pressure loss through the walls as well as through the accumulated soot and deposition layer. In [7] hot film anemometry measurements of the flow downstream of loaded and unloaded DPFs have been performed and substantial non uniformities have been found. Although increasing soot load as well as the presence of an upstream oxidation catalyst (DOC) tend to reduce nonunifomities, these are still evident in the downstream of the DPF, according to [7]. On the other hand reference [8] identifies homogeneous soot loading distribution as a key factor for a reliable DPF system. Nevertheless apart from the flow field factors that create some degree of nonuniformity, modes of soot deposition, volatile organic fraction adsorption and desorption as well as stochastic regeneration phenomena affect the flow, particle, ash and thermal load distribution. Inhomogeneous soot distribution cause locally high thermal stresses during regeneration and can initiate partial regenerations, [9]. These prematurely stopped regenerations lead inevitably to difficulties in determination of the soot load of the DPF based on the pressure drop. Characteristic are the data given in [10 and 11]: Pressure drop of $150 \mathrm{mbar}$ over the filter correspond to a soot load of $0.7 \mathrm{gr}$, when loading an empty filter, while to a soot load of $7 \mathrm{gr}$ following a partial regeneration. Partial regeneration impose, in addition, more severe difficulties to any algorithms aiming to compute the soot load, [12 and 13].

Homogeneous exhaust flow is not only for three way catalysts and diesel particulate filters highly advantageous. In the case of $\mathrm{NO}_{x}$ adsorbers, inhomogeneities will result in inhomogeneous loading and short regenerating periods evoking a higher fuel consumption penalty. In combination with SCR catalysts inhomogeneities will cause inhomogeneous conversion of the reducing agent (ammonia). While regions with ammonia shortage in the reducing catalyst will result in diminished $\mathrm{NO}_{x}$ conversion, regions with ammonia abundance will result in ammonia slip.

Some attempts to achieve homogeneous flow distribution of the exhaust gases upstream of aftertreatment devices have been recently presented. The use of helicaltype flow elements is proposed in [4 and 9]. A progressive spin is imposed by these elements on the exhaust flow upstream of the DPF enhancing mixing. Experimental investigations confirmed that the use of a correctly dimensioned spin element resulted in significantly shorter regeneration phases. A further recent approach concentrated on metal foams, [14]. Metal foams have been tested as particulate filters, [14], as well as Diesel Oxidation Catalysts [15] with very promising results.

Our approach, as presented in this paper, consists in the use of ceramic foam based substrates as diesel oxidation catalysts as well as gasoline precatalysts. The ceramic based micro foams developed in our laboratory (patent applied) are a promising concept, since they redistribute the flow of exhaust gases enhancing turbulence and species mixing, without increasing flow resistance and pressure drop to prohibitive levels. They can initiate, facilitate or even complete oxidation of unburnt $\mathrm{HCs}$ and $\mathrm{CO}$, oxidize a substantial fraction of particulates while generating required species for the following aftertreatment step. Ceramic foams constitute a new structural material characterized by high porosity and high surface area, formed by pores interconnected by filaments $[16,17]$. Having too high flow resistance, [18, 19], to be used directly as particulate filters, [20], their potential lies in precatalyst applications. The turbulent flow inside the ceramic foams allows short overall lengths for high conversion rates. An additional important aspect is the relative low material and manufacturing cost.

In this paper we present main material development aspects as well as flow field investigations performed in the up and the downstream of the ceramic foams, demonstrating their potential.

\section{EXPERIMENTAL SET-UP}

\section{TEST FLOW RIG}

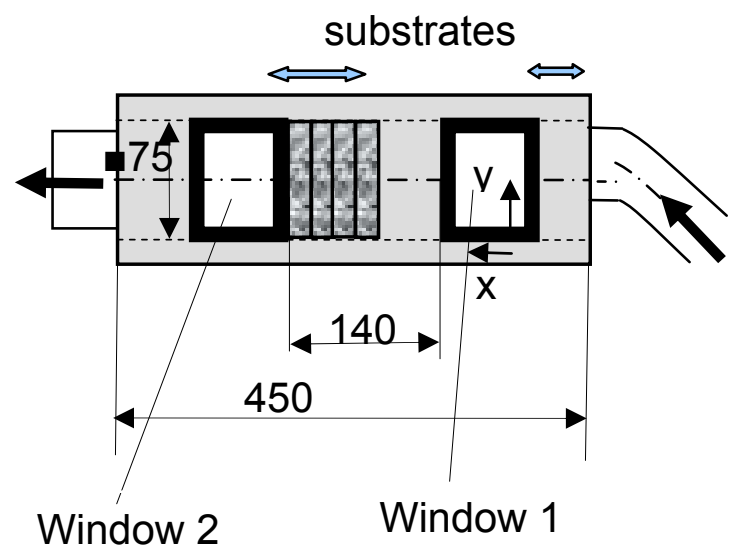

Fig. 1: Schematic of the measurement section of the test flow rig. Also shown are the definitions for the main coordinates. The denoted positive directions correspond also to the assumption for the positive direction of the velocity components.

For the investigations a specifically designed test flow rig has been employed, capable of reproducing exhaust flow conditions typical for passenger cars and light trucks. As a flow medium we used the pressurized air 
circuit of our facilities, providing a wide range of air quantities at ambient temperature and 7 bar feed pressure. Upstream of the measurement section the installation includes a pressure reduction station, mass flow metering, and a 400lt flow oscillation and noise dampener. For the mass flow measurement a hot film anemometer, Type ABB Sensyflow P was used.

The measurement section is modular and consists of the inflow and outflow part, the main channel with two optical access areas as well as two areas for the substrate placement (Fig. 1). The inflow part can easily be changed allowing the assessment of the impact of various automotive exhaust type configurations on the flow upstream and downstream of the substrates. In this paper we report flow studies with only one inflow configuration, eccentric by $10 \mathrm{~mm}$ in respect to the main channel axis and inclined by $45^{\circ}$ (as shown in the schematic of Fig. 1).

The main channel is such that after mounting the substrates with the necessary sealing mat the flow cross section is square with $75 \mathrm{~mm}$ side by side dimensions (Fig. 1). Also in Fig.1 we show the two substrate placement areas. The first area directly after the inflow provides the possibility of mounting substrates of up to $60 \mathrm{~mm}$ length, while the second between the two optical access areas provides the possibility of mounting substrates of up to $140 \mathrm{~mm}$ length. The substrate mounting was performed with a typical automotive sealing mat (3M Interam ${ }^{\mathrm{TM}}$ 100HD Mat).

The optical access areas 1 and 2 consist of four quartz glass windows on each side of the square channel. The measurement equipment contains a number of pressure and temperature sensors at different locations. The setup allows stable metering of flows among 50 and $500 \mathrm{~kg} / \mathrm{h}$ of ambient temperature air resulting in Reynolds numbers between $10^{\prime} 000$ and $100^{\prime} 000$. The kinematic viscosity of exhaust gases is 3-5 times higher (depending on the exhaust gas temperature and composition) than of the ambient air used in this study. Thus results from the investigated mean flows correspond to the behaviour of exhaust flows typical of a mid seized passenger car engine. Pressure drop results maybe directly conveyable to real engine exhaust gas flows.

\section{PARTICLE IMAGE VELOCIMETRY (PIV)}

The PIV technique was chosen for the flow field measurements. The most important advantages of the PIV technique are the abilities to capture planar flow field information and two velocity components simultaneously. The available equipment posed also

two restrictions: No 3-d information could be acquired, while the flow field measurement repetition rate could not exceed $3 \mathrm{~Hz}$.

As a light source we used two independent optically coupled, frequency doubled Nd:YAG-Lasers. The beam followed an optical path mainly consisting of two cylin- drical lenses $(-75 \mathrm{~mm}$ and $1000 \mathrm{~mm}$ focal lengths) resulting in an approx. 400um thin laser sheet in the measurement area. The triggering devices allowed minimal time delays between the two laser shots of $4 \mu \mathrm{s}$, sufficient for the measurement of instantaneous flow velocities of over $70 \mathrm{~m} / \mathrm{s}$ (in combination with the camera lens used). $\mathrm{TiO}_{2}$ particles of an approximate nominal diameter of $1 \mu \mathrm{m}$ have been dispersed in the flow upstream the inflow area of the measurement section. The dispersion was carried out by a home-made solid-powder atomizer.

Perpendicular to the laser sheet a $1280 \times 1024$ pixel CCD camera was mounted. The diameter of each CCD pixel has been $6.7 \mu \mathrm{m}$. The camera lens used, had a $75 \mathrm{~mm}$ focal length and an f/1.19 number resulting to a magnification factor of 0.118 , hence $56.8 \mu \mathrm{m} /$ pixel. The camera was operated in the so-called double exposure mode; following an external trigger two consecutive images had been acquired. High $f$ numbers of the camera have been used during measurements, resulting in a very narrow domain of sharp images and achieving a good spatial filter reducing optical noise.

The processing was performed with the cross correlation method assuming uniform flow distribution in each so called interrogation spot areas. The optimal interrogation spot size was found to consist of $32 \times 32$ pixels corresponding to real square areas of $214 \times 214 \mu \mathrm{m}^{2}$.

The evaluation of each double exposure resulted in the instantaneous flow field. For each measurement configuration around 1500 double exposures have been captured and evaluated. The resulting information was averaged, leading to one mean velocity vector for each $32 \times 32$ pixel area (or for each $214 \times 214 \mu \mathrm{m}^{2}$ area). The standard deviation of all velocity vectors at one pixel from the corresponding mean value was used as the measure of the turbulence intensity at this pixel.

In order to quantify the homogeneity of the flow field we introduced the flow Uniformity Factor (UF) according to [21],

$$
U F=1-\sqrt{\frac{1}{n} \sum_{n}\left(\frac{u_{i}-\bar{u}}{\bar{u}}\right)^{2}}
$$

We computed for each axial velocity profile the uniformity factor in order to be able to follow the evolution of the UF along the flow (with or without ceramic foams). The expression is formulated in such a way that in the case of a completely uniform distribution, UF takes the maximum value of 1 . In the literature the UF is sometimes also called maldistribution factor [7], uniformity index, [21] or $\mathrm{y}$-factor, [5].

All velocities, mean velocities as well as turbulence intensities have been analyzed in one longitudinal (=axial, $x$-direction) and one lateral (=transversal, y-direction) component according to the notions in Fig. 1. The spatial coordinates in Fig. 1 designate also the assumptions for the positive directions of the velocity components. For more details concerning flow measurement techniques 
or the measurement configurations used, the interested reader is referred to [22] and [23].

\section{CERAMIC FOAM SUBSTRATES: MATERIAL DEVELOPMENT ASPECTS}

Ceramic foams are well established filtering materials in the foundry industry. New possibilities open up with so called open porosity ceramic reticular foams enabling porosities of over $90 \%$ and high surface area formed by pores interconnected with filaments. The ceramic foams exhibit relatively low flow resistance while the contacts among the fluid and the filaments are very high. The reticular structure is capable of homogenising nonuniform flows as well the concentration of species in the flow, [24].

Raw materials for such ceramic foams are open, reticular polymer foams. For automotive exhaust aftertreatment applications open-pore ceramics of different compositions produced via the replication of a polymeric porous structure are most suitable, [25, 26, 27]. A first step consists of coating these foams by a ceramic material.

The present application used 10ppi polyurethane foams (roughly corresponding to $100 \mathrm{cpsi}$ ) and alumina $\left(\mathrm{Al}_{2} \mathrm{O}_{3}\right)$ as the coating material. The following consisted in rolling and heat treatment. Rolling important for forming the appropriate surfaces resulted also in blocking several pores reducing the flow cross sectional area. Therefore a specific additional sieving step had to be developed. The heat treatment thereafter is important for sintering the material, but results also in removing the polyurethane basic structure. The cores of the filaments, also called bridges are hollow after the heat treatment (Fig. $3)$.

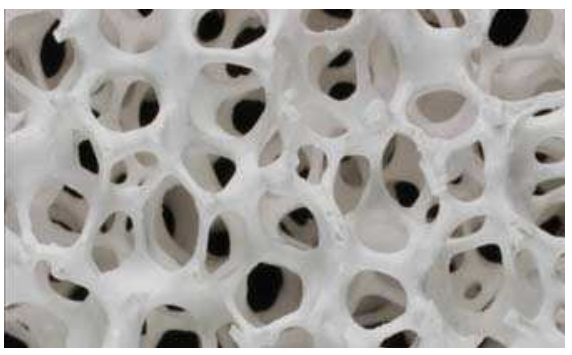

Fig. 2: Enlarged view of an alumina $\left(\mathrm{Al}_{2} \mathrm{O}_{3}\right)$ ceramic foam

The next development steps involved the enhancing of mechanical strength while trying to maintain high surface values. High surface values are crucial for the good performance of a catalyst. Sufficient surface after the sieving and heat treatment process can lead to avoidance of the extra coating step for surface enhancement. Various optimization steps resulted in a trade-off between mechanical strength and surface area. Using fine grained alumina $\left(\mathrm{Al}_{2} \mathrm{O}_{3}\right.$ CT3000SG) and high processing temperatures $\left(1400^{\circ} \mathrm{C}\right)$ the achieved mechanical strength was acceptable, while the specific surfaces below $5 \mathrm{~m}^{2} / \mathrm{g}$ (due to sintering phenomena during the high temperatures). Lower process temperatures resulted in significant higher surface values but very low mechanical strength. Though the resulting surface values of approx. $5 \mathrm{~m}^{2} / \mathrm{g}$ are low, they are high enough for the adhesion of an additional wash coat layer. Nevertheless the achieved higher mechanical strength was still not at a satisfactory level.

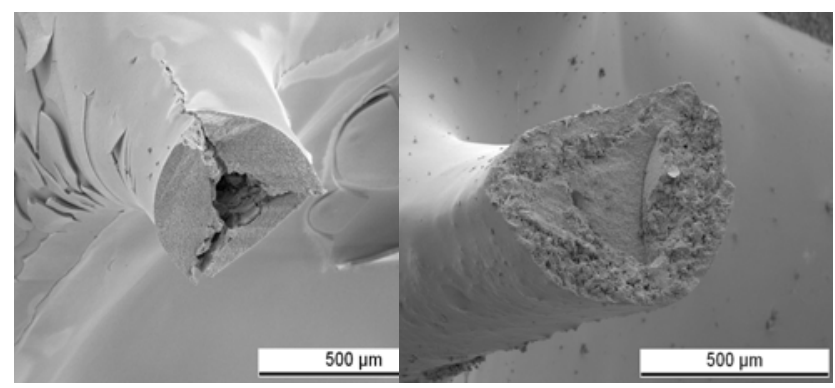

Fig. 3: Scanning electron microscopy (SEM) images of the filaments before (left) and after infiltration (right)

Additional treatment for enhancing mechanical properties was necessary, avoiding further sintering (which would further reduce the available surface). Best results have been achieved by the infiltration of the hollow bridges (Fig. 3). The mechanical properties after the infiltration improved dramatically and reached comparable values of $900 \mathrm{cpsi}$ honeycomb catalyst substrates. For the infiltration several materials have been tested, while best results have been obtained with the same alumina as for the coating $\left(\mathrm{Al}_{2} \mathrm{O}_{3}\right.$ CT3000SG), [25].

The next step, in a proof of concept sense, aimed in assessing the ability of applying a temperature stable wash coat. Various aluminum oxide mixtures have been tested. A detailed description of the results exceeds the scope of this paper.

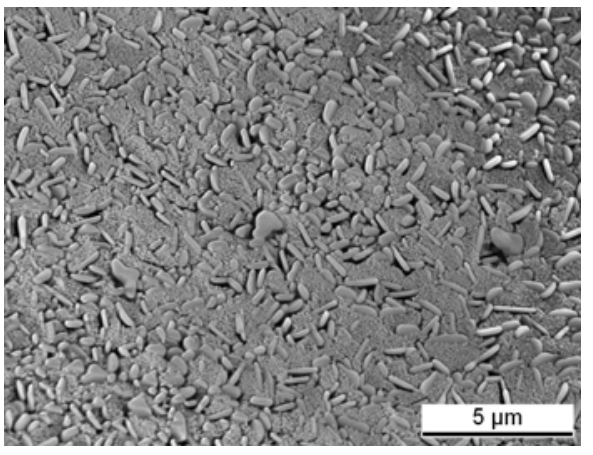

Fig. 4: Surface SEM image of a bridge with boehmit based wash coating after heat treatment of $8 \mathrm{~h}$ at $900^{\circ} \mathrm{C}$.

The resulting ceramic foams have a BET surface exceeding $250 \mathrm{~m}^{2} / \mathrm{g}$ and a temperature stable wash coat layer. Best results have been achieved using Boehmit, an aluminum hydroxide in the $\gamma$-crystallization structure as a wash coat material. Oven heating of approx. $8 \mathrm{~h}$ at $900^{\circ} \mathrm{C}$ only minor affected surface structure, as could be 
seen by SEM images (Fig. 4) or by reapplied BET surface measurements. Fig. 5 shows an application-ready ceramic foam catalyst.

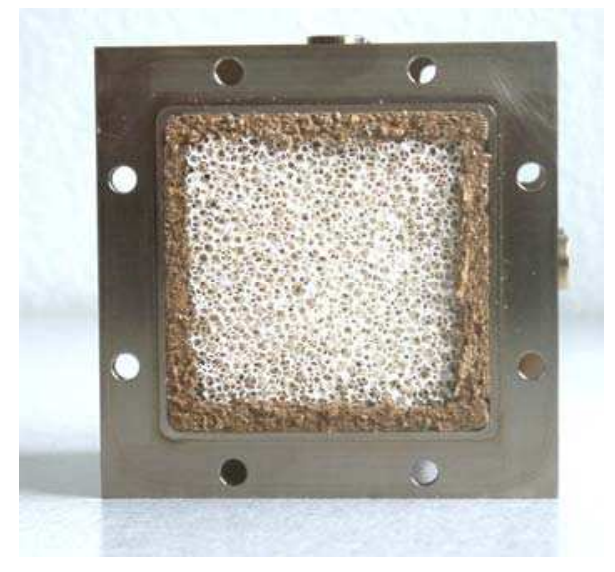

Fig. 5: Image of an application-ready ceramic foam substrate.

\section{RESULTS AND DISCUSSION}

\section{FLOW FIELD INVESTIGATIONS}

Comparison of the flow field upstream and downstream of (80mm long) ceramic foam substrates

In this subsection we compare the flow field upstream and downstream of 4 ceramic foams (approx. $80 \mathrm{~mm}$ of length) placed in the mid of the measurement section (Fig. 1). Thus the comparisons are between the flow fields measured through the optical access areas 1 and 2. All measurements under discussion in this subsection have been performed in the midsection of the square flow channel $(\mathrm{h}=43 \mathrm{~mm})$ and at a mean mass flux of $250 \mathrm{~kg} / \mathrm{h}$.

In Fig. 6 we plot the axial velocity profiles at the extreme locations. The first profile after the entrance in the measuring section (dotted line, wide dots), is located approx. $80 \mathrm{~mm}$ upstream the first ceramic foam. The last profile upstream the ceramic foams (dotted line, narrow dots) is located $35 \mathrm{~mm}$ upstream the first ceramic foam. Similarly the first profile measured after the ceramic foams is located $10 \mathrm{~mm}$ downstream (solid black line) while the last $55 \mathrm{~mm}$ downstream (solid grey line). The direct comparison in Fig. 6 provides evidence for the homogenizing effect of the ceramic substrates. The first velocity profile after the entrance in the measuring section of the channel is highly unsymmetrical. As expected the highest part of the bulk flow is on the right side following the $45^{\circ}$ bend of the inflow section. Flow velocities exceed $25 \mathrm{~m} / \mathrm{s}$ while on the left half of the channel the flow is definitely weaker. The profiles downstream of the ceramic foam substrates are significantly homogenized. Although the comparison in Fig. 6 gives some impression concerning the flow in the channel it has also a misleading aspect; it is obvious that the mean mass flux through the four profiles is not exactly the same. This is primarily due to different measurement sessions in respect of the profiles downstream and upstream of the ceramic foams and inaccuracies in the air metering system. We consider the best comparisons to be among the normalized velocity profiles, computed by dividing the velocities of each profile by the associated mean axial velocity (weighted average across the lateral channel coordinate) as shown in Fig. 7.

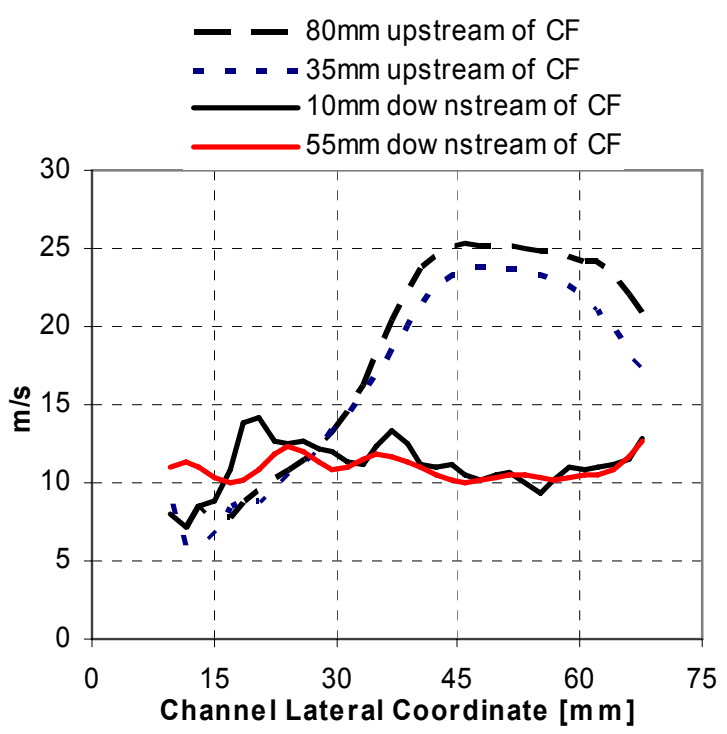

Fig. 6: Mean axial velocity profiles upstream and downstream the ceramic foam substrates $(250 \mathrm{~kg} / \mathrm{h}$ mean flow).

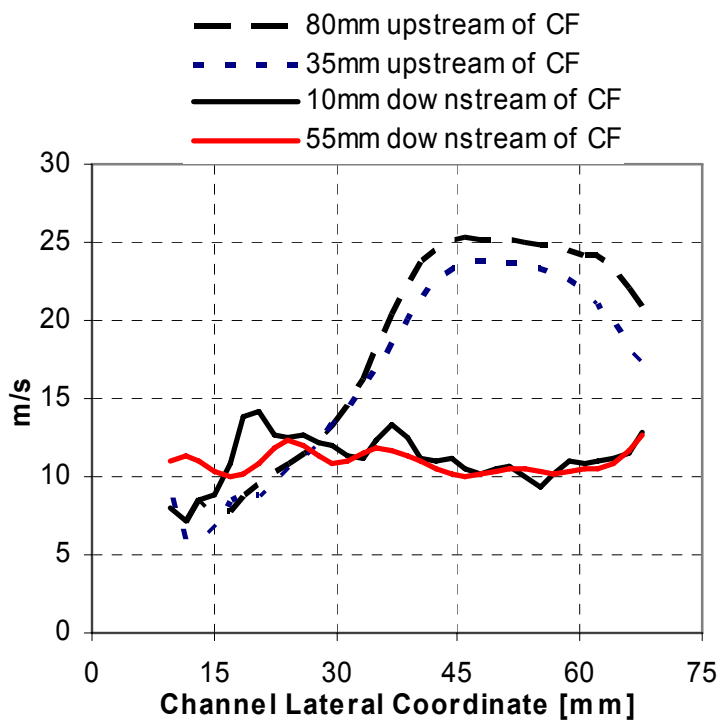

Fig. 7: Normalized mean axial velocity profiles upstream and downstream the ceramic foam substrates $(250 \mathrm{~kg} / \mathrm{h}$ mean flow).

Fig. 7 underlines the homogenizing effect of the ceramic foam substrates. For almost all further plots we will use 
the normalized depiction since it gives a mean mass flow independent view of the phenomena. There is additional evidence that the homogenization of the flow downstream of the ceramic foam substrates is accelerated. This observation is quantified in Fig. 8 (left side) where we plot the flow uniformity factors. Upstream the ceramic foams the uniformity factor is 0.5939 and increases to 0.6303 or by $6 \%$ along the $45 \mathrm{~mm}$ of the measurement plane. This relatively high homogenization rate is due to the strong inhomogeneity and the high strain in the flow caused by the strong lateral velocity gradients. Nevertheless downstream of the ceramic foams the UF of the first profile has reached 0.8612 . Further downstream the UF increases even with a higher rate; approx. $9 \%$ increase (along these $45 \mathrm{~mm}$ ).
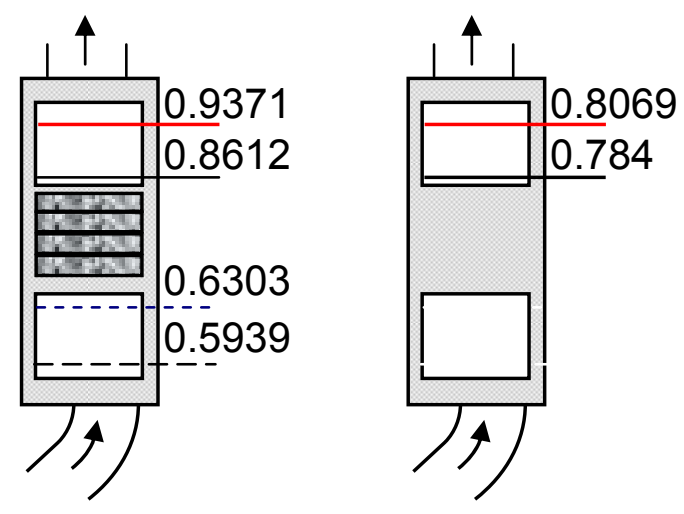

Fig. 8: Flow uniformity (UF) factors upstream and downstream the ceramic foam substrates (left figure) and UFs at the second optical access area without any ceramic foam substrates (channel schematic, no exact scaling).

Without ceramic foams the increase of the UF factor on the exact same area is substantially weaker; as can be seen by the right side of Fig. 8 only a 3\% increase takes place (the comparison of the flow features in the optical access area 2 with and without preceding ceramic foams are subject of separate discussion further below). The uniformity values of the test flow rig upstream of the ceramic foams are of similar order of uniformity factors from real exhaust systems, [7] measured by hot wire anemometry and [5] obtained by CFD simulations.

Acquiring roughly every $0.214 \mu \mathrm{m}$ one mean axial velocity profile, we can compute a mean flux assuming that the velocities are characteristic for an area of $0.214 \times 0.214 \mu \mathrm{m}^{2}$. In addition a simple extrapolation to the entire channel dimensions results in an approximate value for the mean mass flux through each channel cross section. In Fig. 9 we plot the computed mass flux along the channel downstream the ceramic foam substrates. Keeping in mind that the lateral dimension of the measurement area does not cover the entire channel (as can be seen by the velocity profile extensions in Figs 6 and 7 ), the low deviations of the mass flux along the channel, (as can be seen in Fig. 9) provide strong evidence of the quality of the measurement and the evaluation procedure. Similar deviation values have been obtained in all measurements.

The computed mass flux between 247 and $238 \mathrm{~kg} / \mathrm{h}$ lie slightly below the Sensyflow measurement of $250 \mathrm{~kg} / \mathrm{h}$. This is not further surprising since the results are strongly depended on the extrapolation assumptions (the measurement data correspond to one thin slice of the cross section). Extrapolation of the highly nonhomogeneous velocity profiles (upstream of the ceramic foams) resulted in substantially higher mass flux values than those the measured by the Sensyflow.

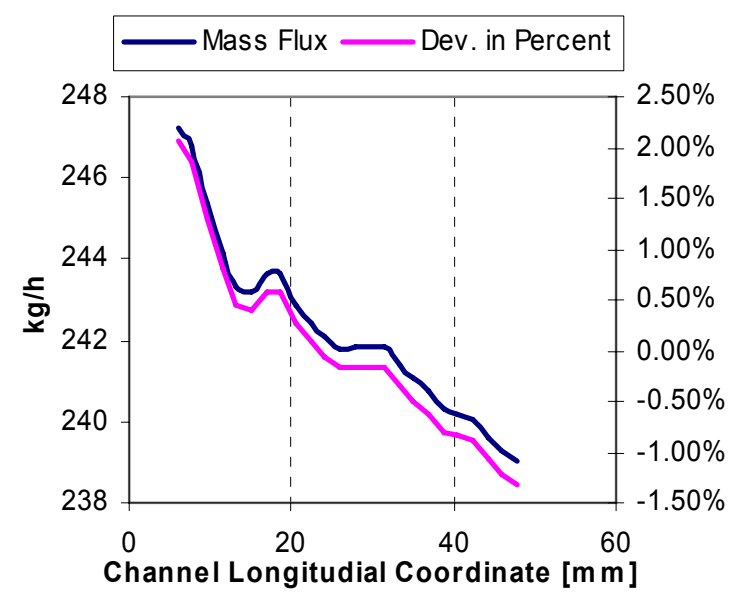

Fig. 9: Mean mass flux along the channel downstream the ceramic foams, computed using the mean flow velocity of each measured, subsequent axial velocity profile.

The axial turbulence intensities showed different behavior upstream and downstream of the ceramic foams, Fig. 10. Upstream of the ceramic foams we have two distinct turbulence areas: The part of high bulk flow (right side of the channel) is characterized by relative low turbulence (below 0.4). The flow field in the other channel side (left side) with very low mean velocities and "inactive" fluid being teared away by the "inlet jet" at the right channel side, shows very high turbulence intensities. Important is also the fact that turbulence increases along the channel. The opposite trend persists downstream the ceramic foams: Turbulence decreases rapidly downstream and it seems that the path through the ceramic foams has a similar effect as a (substantially longer) flow developing section. In addition, downstream the ceramic foams turbulence in the left part $(y<40 \mathrm{~mm})$ of the channel is lower than in the right. Of importance is though the fact that in the right half of the channel $(y>40 \mathrm{~mm})$ turbulence intensities upstream and directly downstream of the substrates are of very similar dimension. Assuming that some turbulence decay must have occured in the $95 \mathrm{~mm}$ between the last profile measured upstream and the first downstream the substrates, then turbulence generation in between must be the case. We consider this fact as evidence for strong turbulence of the flow inside the ceramic foam substrates. 


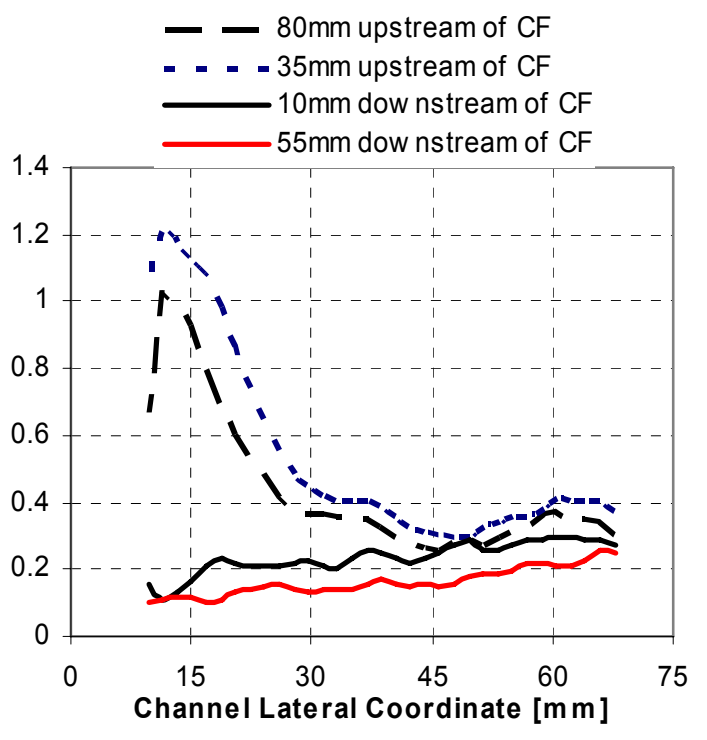

Fig. 10: Normalized axial turbulence intensities upstream and downstream the ceramic foam substrates $(250 \mathrm{~kg} / \mathrm{h}$ mean flow).

Additional insights can be gained by looking at the transversal mean velocities, as shown in Fig. 11. for the locations upstream of the ceramic foams. Again, the normalized depiction is appropriate, performed using the mean axial velocities at the associated coordinates. At $y=15 \mathrm{~mm}$ (the left side of the channel for a downstream observation) the flow is characterized by highly fluctuating, chaotic transversal velocities. Nevertheless, most of these velocities are positive indicating flow motion from the left channel wall to the center of the channel (Fig. 1 shows the assumptions for the positive direction of the velocities).

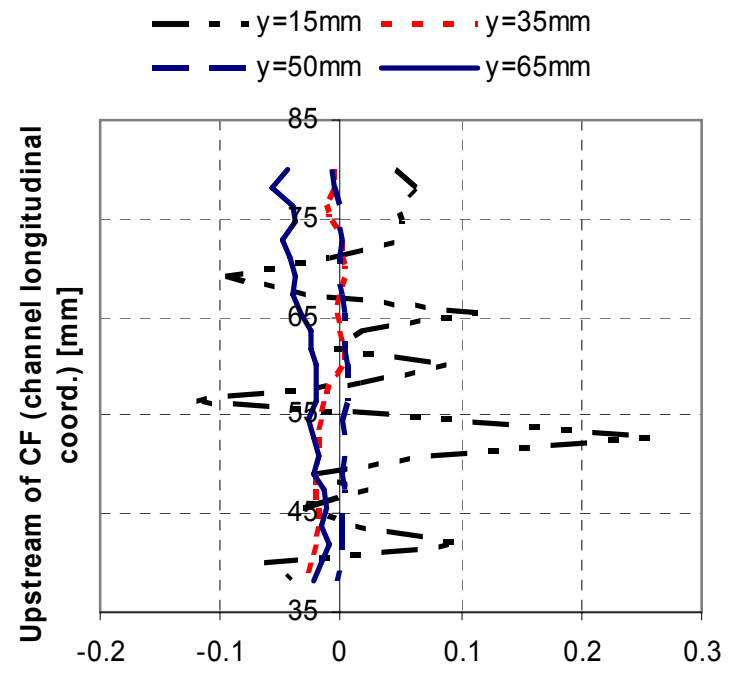

Fig. 11: Normalized mean transversal (=lateral) velocities upstream the ceramic foam substrates $(250 \mathrm{~kg} / \mathrm{h}$ mean flow) at four lateral coordinates.

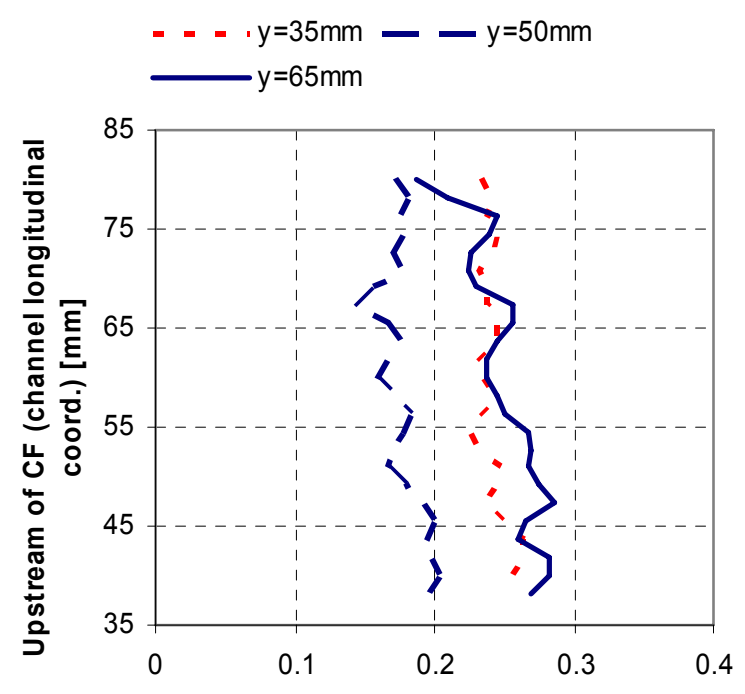

Fig. 12: Normalized transversal (=lateral) turbulence intensities upstream the ceramic foam substrates $(250 \mathrm{~kg} / \mathrm{h}$ mean flow) at three lateral coordinates.

Moving to the other side of the channel transversal fluid motion enfeebles, being extremely low in the channel's midsections and often changing signs. In the right side of the channel some transversal motion is detectable being negative, following, as expected, the expansion of the inlet jet. As expected transversal bulk flow motion is weak, being at least one order of magnitude lower than the corresponding axial.

The associated turbulence intensities on the other hand have very low values at the right side of the channel and increase substantially towards the left side reaching very high values there. Interestingly, transversal turbulence intensities are of the same order as the axial turbulence intensities (Fig. 10). In Fig. 12 we do not include the much higher turbulence intensities at $y=15 \mathrm{~mm}$ (reaching values over 1) in order to be able to resolve the relative magnitude of the turbulence intensities at the coordinates shown. Alike the axial turbulence intensities, transversal turbulence intensities increase along the channel, since the values $35 \mathrm{~mm}$ upstream from the ceramic foams are significantly higher than those $80 \mathrm{~mm}$ upstream from the ceramic foams (Fig. 12).

The confrontation of the transversal flow features upstream and downstream of the ceramic foams yields some more insights. The plots in Fig. 13 correspond exactly to these of Fig. 11, based though on measurement data downstream of the ceramic foams. Apart from the transversal velocities at $y=15 \mathrm{~mm}$, all other mean transversal velocities are higher downstream than upstream the ceramic foams. In addition the velocity characteristics provide some explanation of the homogenization impact of the ceramic foam substrates in their downstream. While in the midsection of the channel transversal velocities are almost zero, on both halves of the channel there is strong transversal motion in the positive 
direction. We consider this a strong evidence for strong mixing processes in the flow. Not surprising, transversal velocities near the right channel wall $(y=65 \mathrm{~mm})$ are weak and negative, pointing towards the channel axis. Nevertheless, the strong lateral mean bulk flow abates in the channel downstream. Values around $10 \%$ of the mean axial velocity still prevail some $45 \mathrm{~mm}$ further in the downstream (Fig. 13).

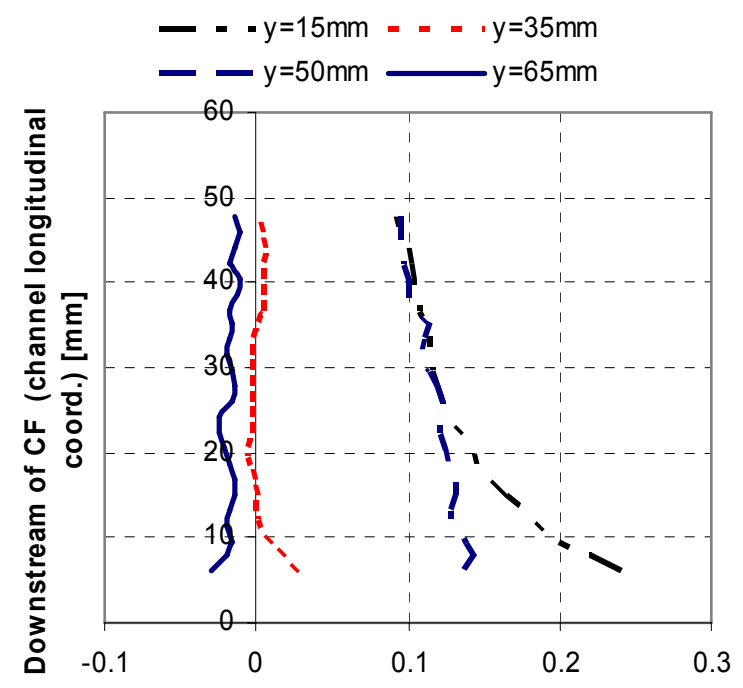

Fig. 13: Normalized mean transversal (=lateral) velocities downstream the ceramic foam substrates $(250 \mathrm{~kg} / \mathrm{h}$ mean flow) at four lateral coordinates.

In Fig. 14 plotted are the normalized transversal turbulence intensities downstream the ceramic foam substrates at 4 coordinates. Most transversal turbulence intensities downstream of the ceramic foams have the same order of magnitude of the corresponding ones upstream of the ceramic foams (except those at the left side, $y<25 \mathrm{~mm}$, of the channel, where "inactive" fluid is being teared away by the "inlet jet" leading to very high turbulence). This is some evidence for a turbulence enhancement through the ceramic foams also in the lateral direction. Further downstream, turbulence decreases reaching in values of around $15 \%$. The turbulence at the two near wall coordinates $(y=15 \mathrm{~mm}$ and $65 \mathrm{~mm})$ is higher than in the location nearer to the channel axis $(y=35 \mathrm{~mm}$ and $50 \mathrm{~mm})$. This can be considered as evidence for the developing effect of the ceramic foams on the flow.

Flow field characteristics at the $2^{\text {nd }}$ optical access area with and without ceramic foams in the upstream

In this subsection the flow field results through the optical access area 2 with the four ceramic foams in their upstream are opposed to the free channel flow in the same area without any ceramic foams. In effect compared are the results of the former section downstream of the ceramic foams to measurements of the flow in the exact same area but with no preceding foam substrates. All measurements shown have been performed in the midsection of the square channel $(\mathrm{h}=43 \mathrm{~mm})$ and at a mean mass flux of $250 \mathrm{~kg} / \mathrm{h}$.

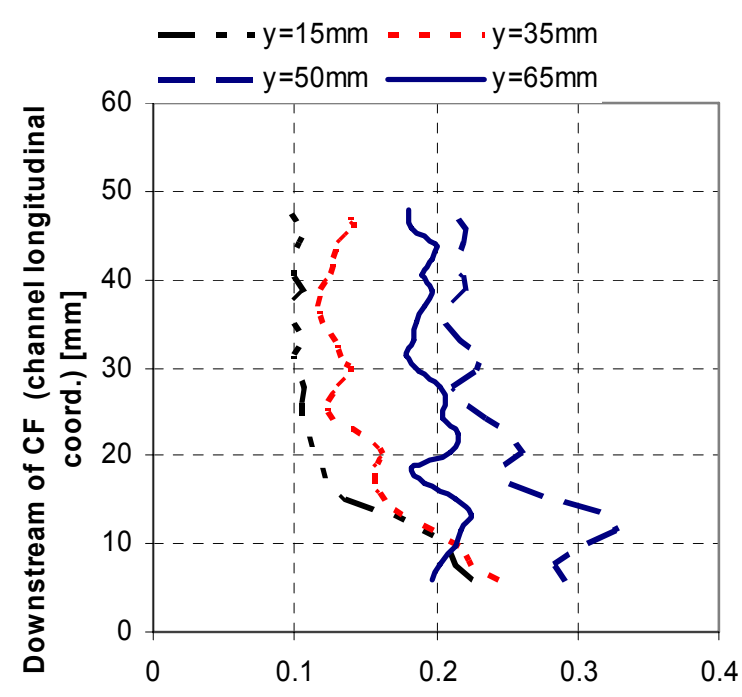

Fig. 14: Normalized transversal (=lateral) turbulence intensities downstream the ceramic foam substrates $(250 \mathrm{~kg} / \mathrm{h}$ mean flow $)$ at four lateral coordinates.

The discussion of Fig. 8, further above, has already compared the uniformity factors (UF) with and without ceramic foams at the same area demonstrating the homogenizing abilities of the ceramic foams. In this subsection the aim is to describe some additional features of the flow field.

The direct comparison of the axial velocity profiles reveals that the inhomogeneous structure of the flow field still prevails in the $2^{\text {nd }}$ flow measurement area in the absence of any ceramic foams (Fig. 15). Though the asymmetries are reduced, the profile is very similar to the profile of the flow in the area upstream the ceramic foams (Fig. 6 and 7).

Axial turbulence intensities in the same area with and without preceding ceramic foams show similar characteristics (Fig. 16), being weaker on the left part $(y<40 \mathrm{~mm})$ of the channel than on the right $(y>40 \mathrm{~mm})$ part. Turbulence decay downstream of the ceramic foam substrates is much more rapid than in the case with no foams. In Fig. 16 axial turbulence intensities in the first measurement location are similar regardless if ceramic foams were there or not. $55 \mathrm{~mm}$ downstream turbulence has strongly decayed in the case of preceding ceramic foams, while without ceramic foams turbulence decay is very weak. This is indicating that the flow path through the ceramic foams acts like a flow developing device or section.

Interestingly, the mean transversal velocities in this $2^{\text {nd }}$ measuring area without ceramic foams in the upstream are almost an order of magnitude lower than in the same area but after the passing through the ceramic foams, as 
can be seen by comparison of Fig. 17 and 13. It is apparent that without the ceramic foams the nonhomogeneous flow will persist for some additional channel diameters in the downstream. It is additionally evident that low mixing occurs lateral to the main flow without the ceramic substrates.

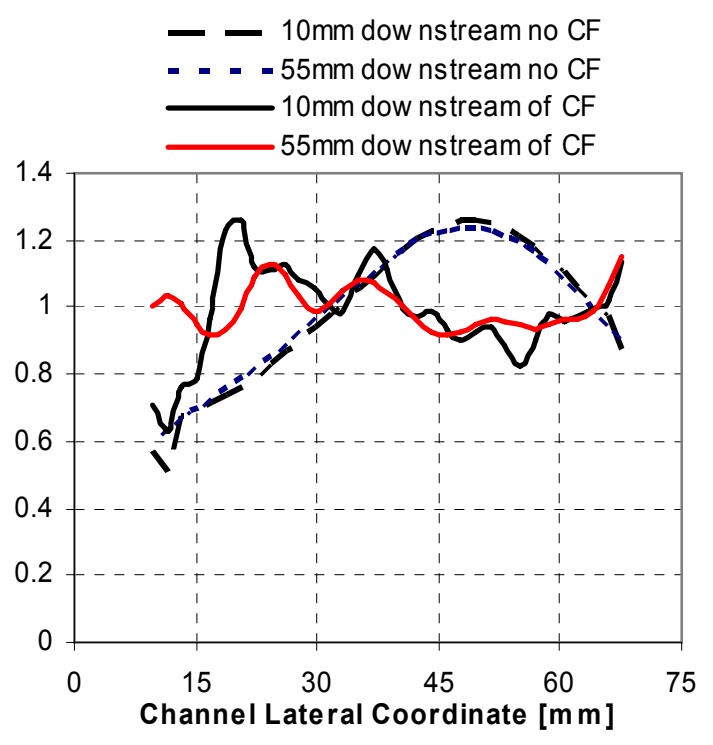

Fig. 15: Normalized axial mean velocity profiles in the $2^{\text {nd }}$ optical access area with and without ceramic foam substrates $(250 \mathrm{~kg} / \mathrm{h}$ mean flow).

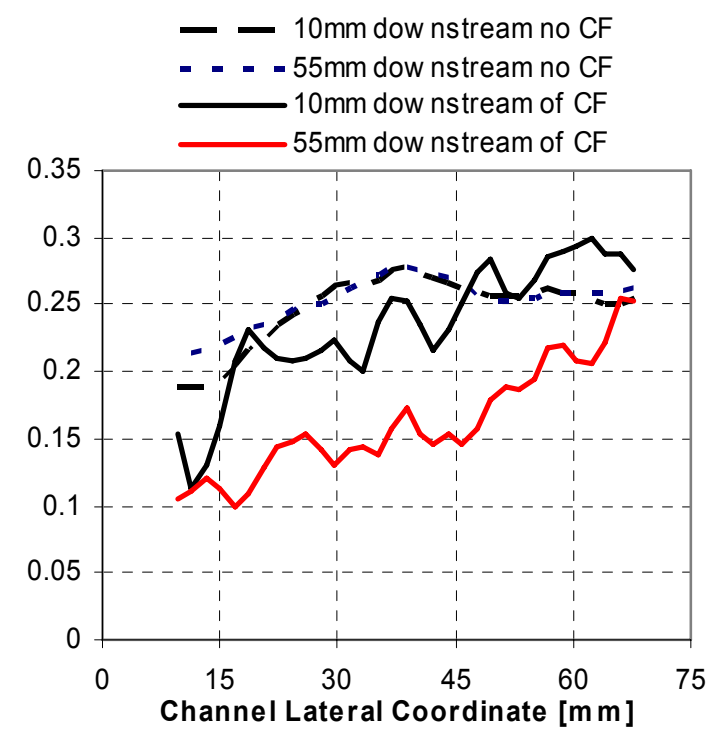

Fig. 16: Normalized axial turbulence intensity profiles in the $2^{\text {nd }}$ access area with and without ceramic foam substrates $(250 \mathrm{~kg} / \mathrm{h}$ mean flow).

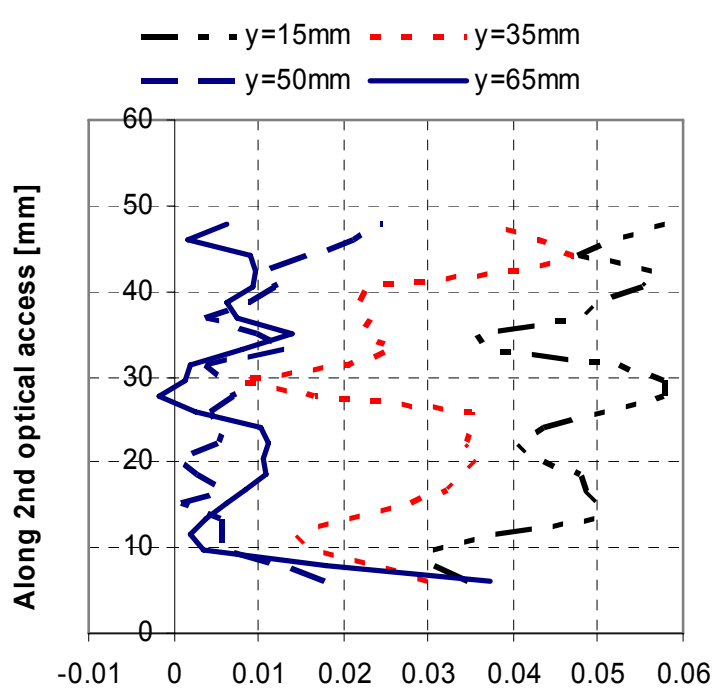

Fig. 17: Normalized transversal mean velocity profiles in the $2^{\text {nd }}$ optical access area without ceramic foam substrates $(250 \mathrm{~kg} / \mathrm{h}$ mean flow $)$ in the upstream at four lateral coordinates.

\section{Influence of the mean mass flow}

All measurements at the different locations have been performed at six different mean mass fluxes, 100, 150, $200,250,300$ and $350 \mathrm{~kg} / \mathrm{h}$. Apart from the time distance between the two laser shots and the two exposures which was adjusted to match the increasing velocity level all other settings have been the same. In the following discussion we will describe the influence of the mean mass flow only at the medial height $(h=43 \mathrm{~mm})$ of the $2^{\text {nd }}$ optical access downstream the ceramic foam substrates. The tendencies in regard of the mean mass flow have been identical at all other measurement positions.

In Fig 18 we plot the normalized mean axial velocity profiles at a chosen location downstream of the ceramic foam substrates for the six different investigated mass fluxes. A specific coarse resolution of the $y$-axis is chosen in order to highlight, if any, the differences. Not surprising, there are no noticeable differences indicating that the mean axial velocity profile is scaling with the mean mass flux. In addition, no mention worthy differences have been found in the normalized mean transversal velocities indicating that comparable scaling accounts also for the bulk flow movement in the lateral direction (in order to limit the length of this paper we omit an associated plot). Largest differences among the profiles are less than $3 \%$. We consider this good agreement as an additional evidence of the accuracy in the application of the PIV technique as well as of the data evaluation procedures.

Nevertheless, turbulence intensities show a different behavior. As can be seen in Fig. 19, lower mass fluxes are characterized by higher, dimensionless, turbulence in- 
tensities. There is factor of more than 3 between the turbulence degree at $100 \mathrm{~kg} / \mathrm{h}$ and $350 \mathrm{~kg} / \mathrm{h}$. These rather surprising results were also confirmed by the turbulence intensities of the transversal velocity component and at all locations. It seems that higher mass fluxes impose a stronger order in the flow allowing only minor fluctuations.

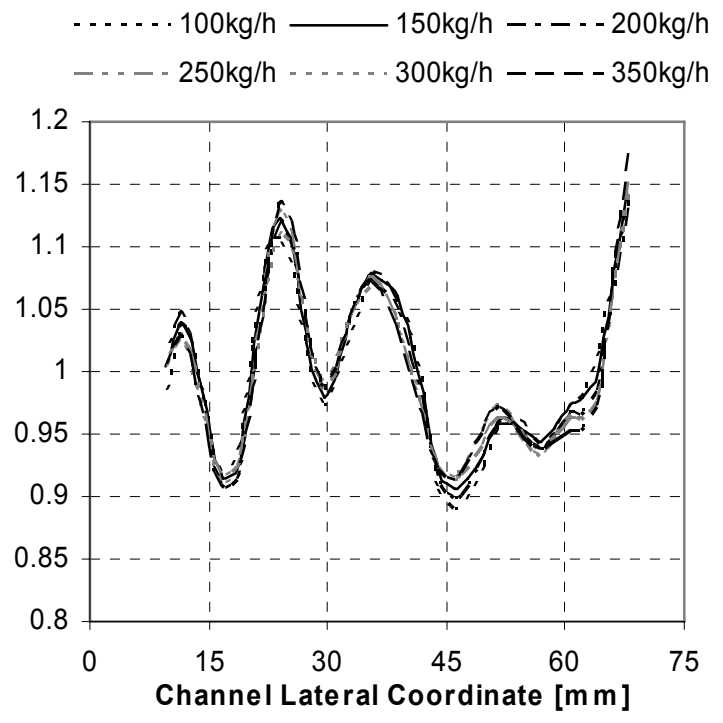

Fig. 18: Normalized axial mean velocity profiles in the $2^{\text {nd }}$ measurement and optical access area downstream the ceramic foam substrates at all measured mean mass fluxes.

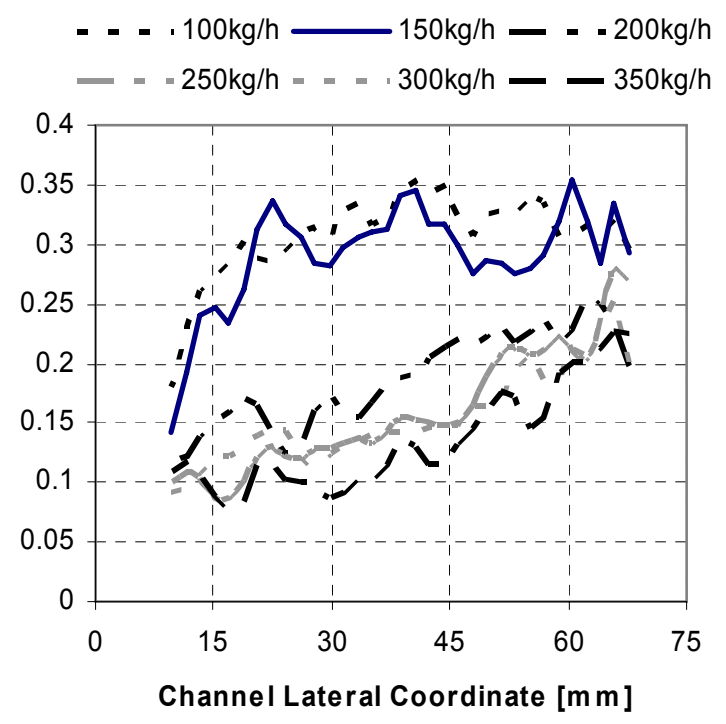

Fig. 19: Normalized axial turbulence intensity profiles in the $2^{\text {nd }}$ measurement and optical access area downstream the ceramic foam substrates at all measured mean mass fluxes.

\section{Flow field comparisons among three heights}

Up to this point all flow field results presented have been measured at a medial height ( $z$ - coordinate) of the chan- nel $(h=43 \mathrm{~mm})$. All different measurements were performed at three heights; the already presented medial height as well as $20 \mathrm{~mm}$ lower and higher. In this subsection compared are the results among these three heights downstream the ceramic foam substrates.

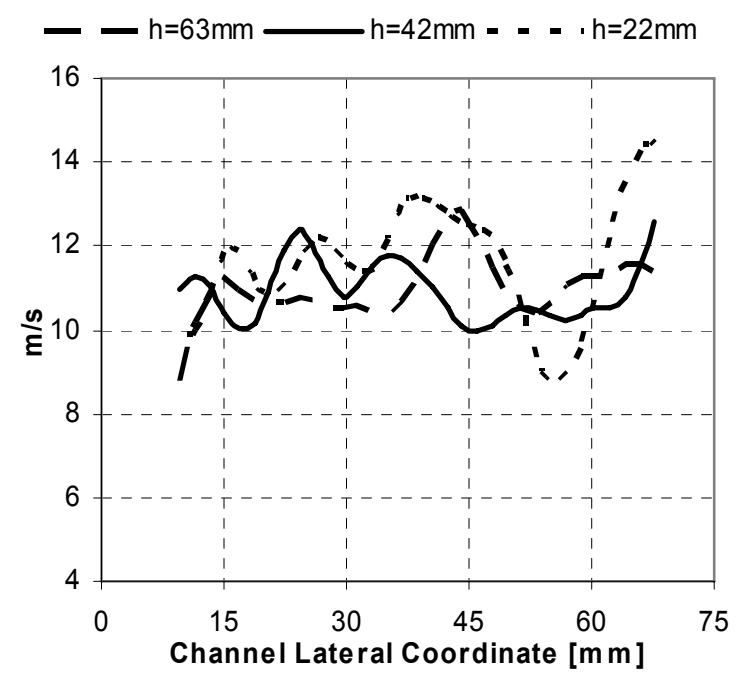

Fig. 20: Axial mean velocity profiles in the $2^{\text {nd }}$ measurement and optical access area downstream the ceramic foam substrates at three heights $(250 \mathrm{~kg} / \mathrm{h}$ mean flow).

The discussion concerns one mean flow, $250 \mathrm{~kg} / \mathrm{h}$. The scaling of the flow properties with the mean flow, as discussed in the former subsection, was evident for all investigated heights. In Fig. 20 we compare the mean axial velocities at the three heights. The velocity profile at the lowest height exhibits the highest inhomogeneities. This may be due to the asymmetric inflow but could also be associated with the structure of the ceramic foam substrates, which may not be as homogeneous as anticipated. Nevertheless, Table 1 shows the uniformity factors for the three heights. As expected lowest values are at $h=22 \mathrm{~mm}$. The issue of the homogeneity of the ceramic foams themselves is important, and next steps in this project will include quantification attempts using computer tomography.

\begin{tabular}{|l|c|c|}
\hline & $\begin{array}{l}\text { 10mm downstream } \\
\text { of CF }\end{array}$ & $\begin{array}{l}55 \mathrm{~mm} \text { downstream } \\
\text { of CF }\end{array}$ \\
\hline $\mathrm{h}=63 \mathrm{~mm}$ & 0.877 & 0.931 \\
\hline $\mathrm{h}=42 \mathrm{~mm}$ & 0.861 & 0.937 \\
\hline $\mathrm{h}=22 \mathrm{~mm}$ & 0.856 & 0.901 \\
\hline
\end{tabular}

Table 1: Flow uniformity factors at the three heights, derived from measurements in the downstream of the ceramic foams at $250 \mathrm{~kg} / \mathrm{h}$ mean flow

In Fig. 21 plotted are the associated axial turbulence intensities. Remarkable is the fact, that turbulence intensity is higher at the peripheral locations. It is even almost 
double as high as in the central location. This is not unexpected, surprising though is, that a similar tendency could not be observed at the perpendicular, lateral channel dimension (y-axis). Figures 10, 14 and 16 show all similar profiles of the turbulence intensities, being low at the one channel side while increasing towards the other.

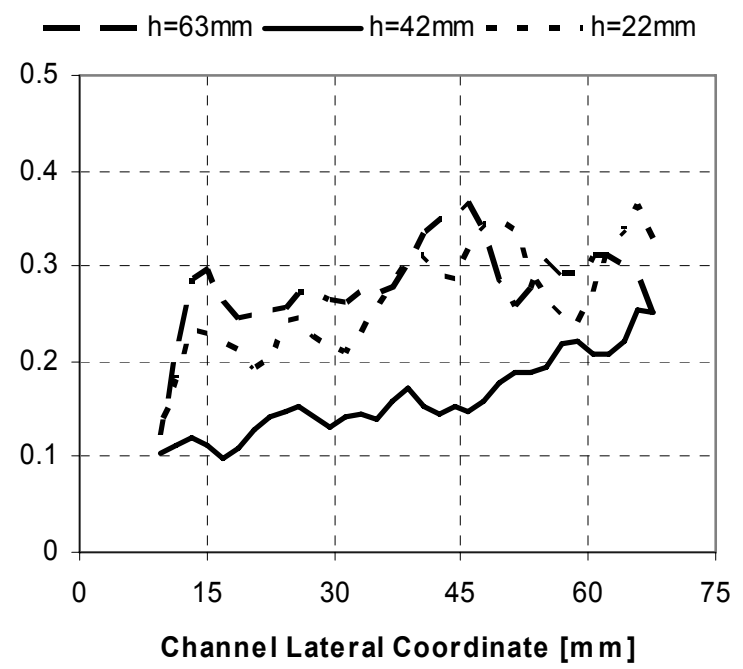

Fig. 21: Axial mean turbulence intensities in the $2^{\text {nd }}$ measurement and optical access area downstream the ceramic foam substrates at three heights $(250 \mathrm{~kg} / \mathrm{h}$ mean flow).

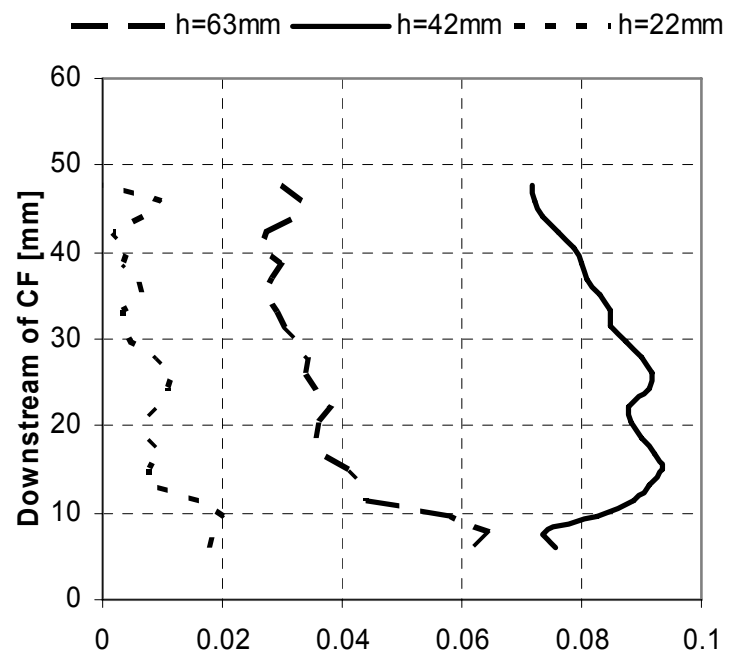

Fig. 22: Normalized transversal mean velocity profiles in the $2^{\text {nd }}$ measurement and optical access area downstream the ceramic foam substrates at three heights $(250 \mathrm{~kg} / \mathrm{h}$ mean flow) and $\mathrm{y}=30 \mathrm{~mm}$.

Fig. 22 shows a comparison of the transversal velocity profiles at the three heights along the channel downstream the ceramic foams. For the comparison a lateral coordinate of $y=30 \mathrm{~mm}$ has been chosen, where phenomena seemed to be representative. Transversal velocities at the lowest measurement height are very low.
With increasing height there is a gradual increase, leading to the highest transversal velocities at approx. $\mathrm{h}=42 \mathrm{~mm}$. Thereafter transversal velocities decrease, still having moderate values at $h=63 \mathrm{~mm}$. Nevertheless, at all three heights the transversal velocities are positive. Almost all transversal velocities downstream the ceramic foams are positive, i.e. at all three heights the bulk fluid movement lateral to the main flow axis is transporting fluid from low to high y-coordinates. This can also be observed on Fig. 13. Fig. 13 shows the transversal velocities at the medial height $(\mathrm{h}=43 \mathrm{~mm})$ for four different $y$-coordinates. Only in the vicinity of the right wall, transversal velocities become negative (this being the case at all three heights), since in the vicinity of the wall, no fluid transport towards the wall is possible.

Not at all y coordinates the relation of the lateral velocities among the three heights was like the one at Fig. 22, the midsection having the higher velocities. At $h=23 \mathrm{~mm}$ transversal velocities are the lowest in comparison to the other two heights. In some other y-coordinates though, the lateral velocities at $h=63 \mathrm{~mm}$ have been higher than at $h=42 \mathrm{~mm}$.

On the other hand the transversal turbulence intensities rather confirmed the behavior of the axial ones (Fig. 21), having high values at the peripheral locations and low in the midsection.

Comparison of the flow field upstream and downstream the ceramic substrates $(30 \mathrm{~mm}$ long), placed directly in the inflow of the measurement section (measurements through optical access area 1)

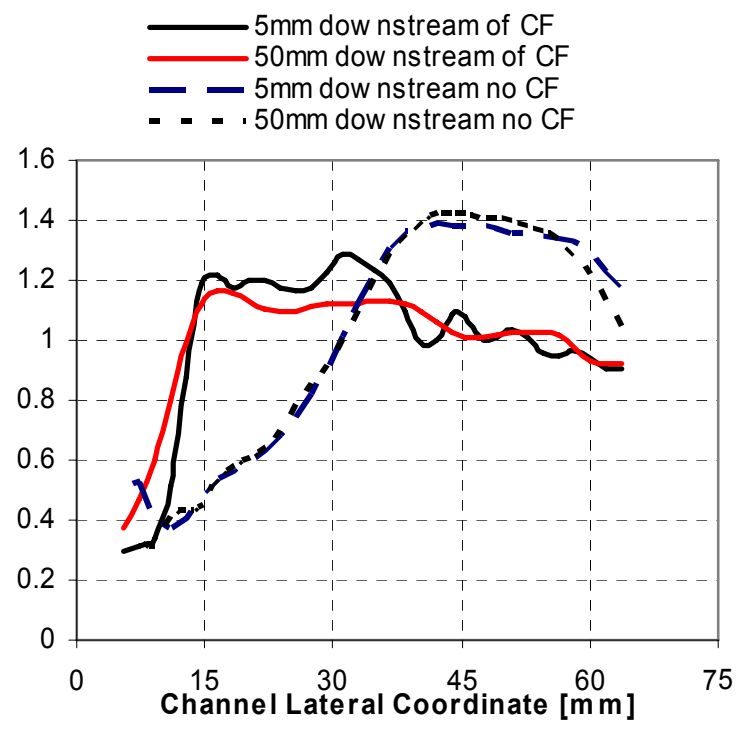

Fig. 23: Normalized mean axial velocity profiles at the optical access area 1 with and without ceramic foams $(250 \mathrm{~kg} / \mathrm{h}$ mean flow).

This additional measurement series aimed in addressing application-near issues. Vehicle exhaust systems are 
compact. Introduction of a ceramic foam as a diesel oxidation catalyst upstream of the particle filter can be mounted directly after the entrance cone of the canned system, only a couple of cms upstream the DPF.
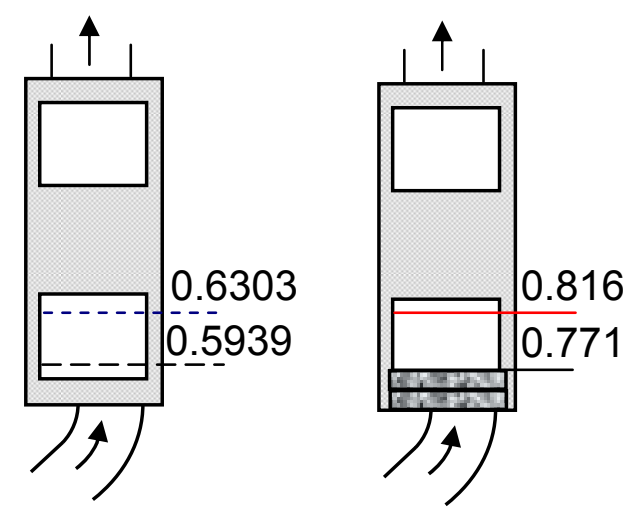

Fig. 24: Normalized mean axial velocity profiles at the optical access area 1 with and without ceramic foams $(250 \mathrm{~kg} / \mathrm{h}$ mean flow).

The homogenising abilities in such a configuration, ceramic foams with significantly reduced length, is the issue of this subsection. Therefore two ceramic foam substrates with a total length of $30 \mathrm{~mm}$ have been placed directly after the inflow. The measurements reported here have been performed at the first optical access area. We restrict our report to the measurements in the channel midsection at a mean flow of $250 \mathrm{~kg} / \mathrm{h}$.

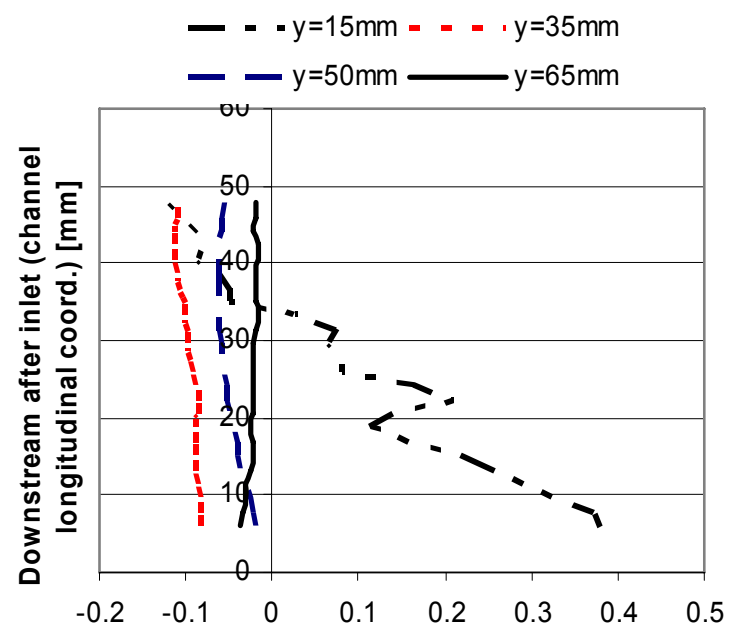

Fig. 25: Normalized mean transversal velocity profiles at the optical access area 1 downstream of the ceramic foam substrates $(250 \mathrm{~kg} / \mathrm{h}$ mean flow).

Fig. 23 and 24 give strong evidence for the homogenizing abilities of the ceramic foams with only $30 \mathrm{~mm}$ of length even placed directly after the unsymmetrical inflow. A comparison with Fig. 8 underlines the results. The homogenization is due to directed lateral bulk flow and this can be supported by the profiles of the transversal velocities (Fig. 25). Once again we observe the lateral bulk motion introduced by the ceramic foams which is directed and significantly larger than the corresponding motion at the same place without ceramic foams (Fig. 11). The high lateral velocities are also evident for enhanced mixing among the exhaust gas components.

\section{FLOW RESISTANCE: THE PRICE TO PAY}

In order to give a most comprehensive comparison of the flow resistance of the ceramic foam substrates we placed in the measuring section ceramic foams of several lengths as well as conventional monolithic honeycomb catalyst substrates of two densities (400 and $900 \mathrm{cpsi}$ ) and different lengths. For the investigations and the results presented here we used the plain substrates without any catalytical or other wash coating.

The flow through the ceramic foams is associated with a higher pressure drop. Taking the $36 \mathrm{~mm}$ length and $400 \mathrm{~kg} / \mathrm{h}$ air flow as a reference we observe $35 \mathrm{mbar}$ pressure loss with the ceramic foams, $23 \mathrm{mbar}$ with the $900 \mathrm{cpsi}$ and $17 \mathrm{mbar}$ with $400 \mathrm{cpsi}$ honeycomb monoliths. Further development of the foams can improve the situation by increasing the overall porosity and decreasing the mean pore size.

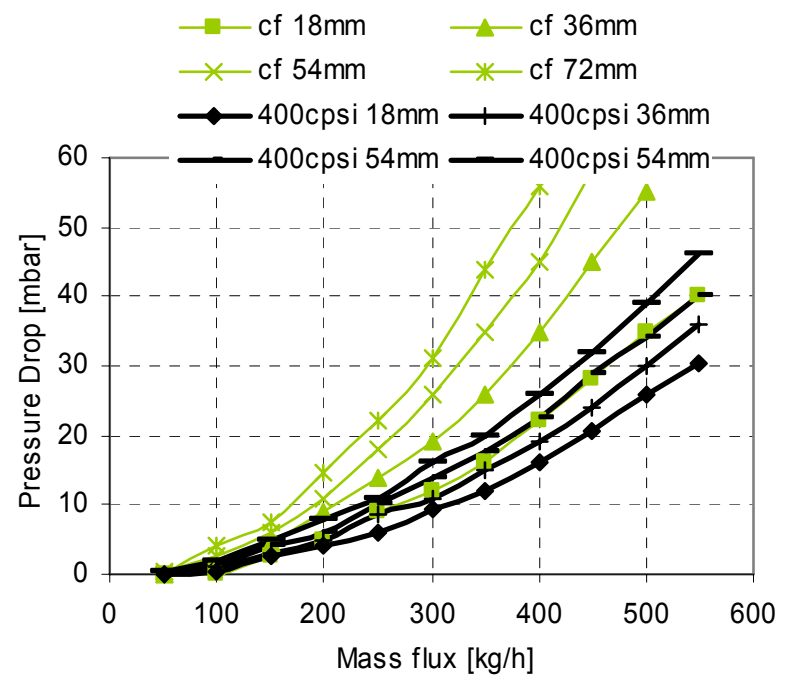

Fig. 26: Pressure Drop of the flow through the ceramic foam substrates vs. the $400 \mathrm{cpsi}$ honeycomb monolithic substrates 


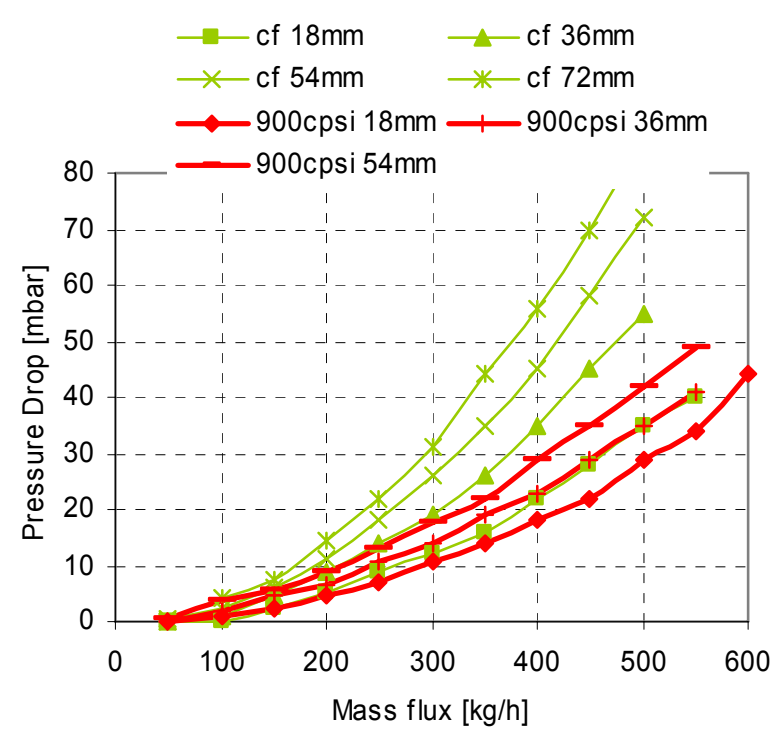

Fig. 27: Pressure Drop of the flow though the ceramic foam substrates vs. the $900 \mathrm{cpsi}$ honeycomb monolithic substrates

\section{CONCLUSIONS AND OUTLOOK}

We have demonstrated the potential of ceramic foams as substrates for catalysts in automotive exhaust gas aftertreatment applications. In a first step we established a relatively simple and cost effective process for producing open pore reticular ceramic foam structures consisting of:

- Coating polyurethane foams with fine grained alumina followed by

- Heat treatment for removing the polyurethane foam of the filaments

- High temperature treatment for a mechanical stable foam structure

- Infiltrating the hollow foam bridges by an alumina slurry and high temperature heat treatment for further enhancing the mechanical properties

These steps lead to

- Comparable mechanical properties with classic honeycomb substrate structures

- Good preconditions for applying a temperature stable high surface area wash coat

The PIV flow field measurements provide numerous evidence for the abilities of the developed ceramic foam substrates to
- homogenize the flow field

- enhance mixing in the flow

- introduce some turbulence

- accelerate flow development

- without prohibitively increasing flow resistance

These results could be repeatedly obtained at a number of mean flow rates, several locations in the channel and with two different dimensions of ceramic foam substrates.

In this paper we provide wide evidence of a series of the advantages of ceramic foams as catalyst substrates. At present, ceramic foam substrates have been catalytically coated by one of the industrial project partners. Further investigations will involve conversion and reactivity studies at the exhaust of CNG engines and direct comparisons with honeycomb monoliths coated with identical washcoat. In a second step the application as a diesel oxidation catalyst is planned upstream of a particulate filter. Tomographic investigations should quantify the impact of the substrate on the particulate filter loading and regeneration behavior.

\section{ACKNOWLEDGMENTS}

Support for this project was granted by

- Competence Center Energy and Mobility (CCEM) of the Paul Scherrer Institute, Switzerland,

- Fiat Powertrain Technologies, Iveco Motorenforschung AG, Arbon Switzerland

- Umicore Automotive Catalysts AG, Hanau-Wolfgang, Germany.

The authors express their gratitude to these institutions as well as to the involved persons.

The authors would also like to express their gratitude to R. Ziegler for his important contribution to all mechanical tasks. We are also grateful to P. Rebecchi for his assistance in various programming and data handling problems as well as to A. Brönstrup and G. Wagner for the production of the ceramic foam substrates.

Last but not least we would like to thank P. Novak for his great assistance in all electronic questions involved in the triggering and synchronizing of lasers and camera. 


\section{REFERENCES}

1. Pattas $\mathrm{KN}$, Stamatelos $\mathrm{AM}$, Pistikopoulos PK, Koltsakis GC, Konstantinidis PA, Volpi E, Leveroni E. Transient modelling of three-way catalytic converters, J. Fuels Lubric. 103 565-578, 1994

2. Zygourakis K. Transient operation of monolithic catalytic converters: A two-dimensional reactor model and the effects of radially nonuniform flow distribution, Chem. Eng. Sci. 44 (9) 2075-2086, 1989

3. Chakravarthy VK, Conklin JC, Daw CS, D'Azevedo. Multi-dimensional simulations of cold-start transients in a catalytic converter under steady inflow conditions, Applied Catalysis A: General 241 289-306, 2003

4. Gaiser G, Oesterle J, Braun J, Zacke P. The Progressive Spin Inlet-Homogeneous Flow Distributions Under Stringent Conditions, SAE Paper 2003-01-0840, 2003

5. Windmann J, Braun J, Zacke $\mathrm{P}$, Tischler $\mathrm{S}$, Deutschmann O, Warnatz J. Impact of the Inlet Flow Distribution on the Light-Off Behavior of a 3-Way Catalytic Converter, SAE Paper 2003-01-0937, 2003

6. Hagelücken C. (editor). Autoabgaskatalysatoren, Tae Expert Verlag, Band 612, 2005

7. Stratakis GA, Stamatelos AM. Flow maldistirbution measurements in wall-flow diesel filters, Proc. Instn Mech. Engrs Vol. 218 Part D: J. Automobile Engineering, IMechE, 2004

8. Ranalli M, Hossfeld C, Kaiser R, Schmidt S, Elfinger G. Soot Loading Distribution as a Key Factor for a reliable DPF System: An innovative Methodology, SAE Paper 2002-01-2158, 2002

9. Oesterle J, Gaiser G, Zacke P. Homogeneous Loading and Regeneration of Diesel Particulate Filters using Progressive Spin Elements, SAE Paper 2004-011424, 2004

10. Gaiser G, Mucha P. Prediction of Pressure Drop in Diesel Particulate Filters Considering Ash Deposit and Partial Regenerations, SAE Paper 2004-01-0158, 2004

11. Gaiser G. Berechnung von Druckverlust, Russund Ascheverteilung in Pertikelfiltern, MTZ 66 92-102, $2 / 2005$

12. Konstandopoulos AG, Skaperdas E, Masoudi M. Inertial Contributions to the Pressure Drop of Diesel Particulate Filters, SAE Paper 2001-01-0909, 2001

13. Hinterberger C, Kaiser R, Olesen M. 3D-Simulation von Russbeladung und Dieselpartikelfilter-Regeneration, MTZ 67 232-241, 4/2006
14. Koltsakis GC, Katsaounis D, Samaras ZC, Naumann D, Saberi S, Böhm A. Filtration and Regeneration Performance of a Catalyzed Metal Foam Particulate Filter, SAE Paper 2006-01-1524, 2006

15. Hossfeld C, Ranalli M. Katalysatorkonzept zur Reduzierung des Dieselpartikelfilter Volumens, MTZ 67 628-635, 9/2006

16. Lange FF, Miller KT. Open-Cell, Low-density ceramics fabricated from reticulated polymer substrates, Advanced Ceramic Materials, Vol. 2, No. 4, pp. 827-831, 1987

17. Vogt UF, Brönstrup A, Thünemann M, Durisch W, Tober W, Graule T. Poröse Yb2O3 Keramikemitter für die Thermophotovoltaik, cfi/Ber DKG 82 No. 13, pp 231-234, 2005

18. Moreira EA, Innocentini MDM, Coury JR. Permeability of ceramic foams to compressibal and incompressible flow, Journal of the European Ceramic Society 24, 3209-3218, 2004

19. Richardson JT, Remue D, Hung JK. Properties of ceramic foam catalyst supports: mass and heat transfer, Applied Catalysis A: General 250 319-329, 2003

20. van Basshuysen R, Schäfer F. Handbuch Verbrennungsmotor, 2. Auflage, 2002

21. Nagel T, Diringer J. Minimum Test Requirements for High Cell-Density, Ultra-Thin Wall Catalyst Supports, SAE Paper 2000-01-0495, 2000

22. Ruck B (editor). Lasermethoden in der Strömungsmesstechnik, AT-Fachverlag $\mathrm{GmbH}$, Stuttgart, 1990

23. Herrmann K. Strömung, Flammencharakterisierung und Stickoxid-Bildung in Turbulenten Vormischflammen, PhD Thesis, ETHZ, 2002

24. Adler J, Standtke G. Offenzellige Schaum-keramik, Part 1 and 2, Keramische Zeitschrift 55, 2003

25. Wagner G. Oberflächenmodifizierte Keramikschäume für die Abgastechnologie, Diploma Thesis Empa and FH Koblenz, 2005

26. Schwartzwaelder K, Sommer AV. Method of making a porous shape of sintered refractory ceramic articles, United States Patent No. 3090094, 1963

27. Vogt UF, Gyoerfy L, Herzog A, Graule T, Plesch G. Macroporous Silicon Carbide Foams for Porous Burner Applications and Catalyst Supports, Journal of Physics and Chemistry of Solids, Special Issue SSC 2006 (in press) 


\section{CONTACT}

P. Dimopoulos, Dr. sc-techn., Empa,

Swiss Federal Laboratories for Materials Testing and Research, Laboratory for I.C. Engines,

Ueberlandstrasse 129

$\mathrm{CH}-8600$ Duebendorf, Switzerland,

Tel.: +41-44-8234337, Fax: +41-44-8234041,

email: panayotis.dimopoulos@empa.ch

\section{DEFINITIONS, ACRONYMS, ABBREVIATIONS}

\begin{tabular}{|l|lll|}
\hline \multicolumn{4}{|l|}{ Abbreviations } \\
\hline BET & Brunau Emmett & Teller surface \\
\hline
\end{tabular}

\begin{tabular}{|l|l|}
\hline & measuring technique \\
\hline CF & Ceramic Foams \\
\hline cpsi & Cells per square inch \\
\hline DOC & Diesel Oxidation Catalyst \\
\hline DPF & Diesel Particulate Filter \\
\hline ppi & Pores per inch \\
\hline SEM & Scanning Electron Microscopy \\
\hline TWC & Three way catalyst \\
\hline UF & Uniformity Factor \\
\hline Latin characters \\
\hline $\mathrm{x}$ & $\begin{array}{l}\text { Direction along main flow veloc- } \\
\text { ity (=axial velocity) }\end{array}$ \\
\hline $\mathrm{y}$ & $\begin{array}{l}\text { Direction perpendicular to the } \\
\text { main flow velocity (=lateral or } \\
\text { transversal flow velocity) }\end{array}$ \\
\hline
\end{tabular}

